variant consistent with a diagnosis of COL4A1-related disorder. Genetic testing of the parents showed that mum had low level mosaicsm, approximately $27 \%$ in leukocyte DNA for the COL4A1 Missense variant.

Conclusion This is the first case of COL4A1 related disorder due to mosacism. Prevalence of COL4A1-related disorder are difficult to establish as fewer than 100 families have been described in the literature. It is likely they are underestimated because of their multisystem and variable phenotype. The significance of the mosaic variant is unknown and mum will be followed up in the adult neurology services to assess for both clinical and radiological features suggestive of subclinical disease.

\section{GP236 FETAL SURGERY FOR SPINA BIFIDA A NEW DEVELOPMENT IN THE IRISH SETTING}

${ }^{1}$ John Joyce*, ${ }^{2}$ Sieglinde Mullers, ${ }^{2}$ Fergal Malone, ${ }^{1}$ Michael Boyle. ${ }^{1}$ Department of Neonatology, Rotunda Hospital, Dublin, Ireland; ${ }^{2}$ Department of Fetal Medicine, Rotunda Hospital, Dublin, Ireland

\subsection{6/archdischild-2019-epa.295}

Introduction Neural tube defects occur in approximately 1/1000 live births in Ireland, with myelomeningocele being the most common form. The current standard management in Ireland involves postnatal closure however access to fetal surgery services is increasing. The Management of Myelomeningocele Study (MOMS) compared prenatal and postnatal closure and showed reduced risk of death, decreased need for ventriculo-peritoneal shunting and improved motor function in the prenatal closure group. We present a case of fetal closure of myelomeningocele referred from our institution.

Clinical Case A 33-year-old female presented to the Rotunda for antenatal care in her second pregnancy following in vitro fertilisation abroad. Her first child was born with myelomeningocele and is a wheelchair user. She had been taking folic acid before and throughout this pregnancy. Antenatal ultrasound revealed a lemon-shaped head with Arnold-Chiari malformation, ventriculomegaly and myelomeningocele at L5/S1. Karyotype and microarray from amniocentesis were normal. She was referred to UCH London for fetal surgery consideration and ultimately underwent hysterotomy and closure of fetal myelomeningocele S1-S3 in Leuven, Belgium at 26 weeks gestation. The procedure involved successful closure of the dura and fascial layers, however, primary closure of the skin was not possible and so a silicone dermal regeneration template was applied to the defect. Improvement in head shape, ventricular size and Chiari malformation were noted on subsequent fetal ultrasound throughout the pregnancy.

The patient presented with abdominal pain at $35+5$ weeks gestation and proceeded to caesarean section. A live born male weighing $3.1 \mathrm{~kg}$ was delivered. He was vigorous with good lower limb movements and anal tone. The silicone graft was in position on the sacrum at delivery and peeled away spontaneously revealing a $2 \mathrm{~cm}$ by $2 \mathrm{~cm}$ patch of healthy granulating skin. There was no defect or leakage of cerebrospinal fluid. A silicone based wound dressing was applied and the infant was admitted to NICU for management. Enteral feeds were quickly established and postnatal cranial ultrasound was normal. He was transferred to Children's University Hospital for ongoing multidisciplinary team input.

Discussion This case represents the second patient to undergo fetal surgery for spina bifida from our institution, in a new partnership with UCH London and Leuven, Belgium. While the surgery is associated with risks of preterm delivery, intraoperative complications and uterine rupture, the potential for significantly better postnatal outcomes make it an important treatment option for some families and should be considered for discussion in suitable cases as they arise.

\section{GP237 THE USE OF MASSAGE THERAPY FOR TREATMENT OF NEONATAL HYPERBILIRUBINEMIA: A SYSTEMATIC REVIEW AND NETWORK META-ANALYSIS}

${ }^{1}$ Mohammed Abdelrahman*, ${ }^{2}$ Nguyen Lam Vuong, ${ }^{3}$ Gehad Tawfik, ${ }^{4}$ Do Phuc Nhu Nguyen, ${ }^{5}$ Le Van Thanh, ${ }^{6}$ Muhammed Elfaituri, ${ }^{7}$ Marwa Mansour, ${ }^{2}$ TB Thoa Le, ${ }^{8}$ Marwa Zaki, ${ }^{9}$ Kenji Hirayama, ${ }^{9}$ Nguyen Tien Huy. ${ }^{1}$ Al-Adan hospital, Kuwait, Kuwait; ${ }^{2}$ University of Medicine and Pharmacy at Ho Chi Minh City, Ho Chi Minh City, Vietnam; ${ }^{3}$ Faculty of Medicine, Ain Shams University, Cairo, Egypt; ${ }^{4}$ Epidemiology Department, The Institute of Public Health Ho Chi Minh City, Ho Chi Minh City, Vietnam; ${ }^{5}$ Faculty of Medicine, University of Medicine and Pharmacy at Ho Chi Minh City, Ho Chi Minh City, Vietnam; ${ }^{6}$ Faculty of Medicine, University of Tripoli, Tripoli, Libya; ${ }^{7}$ Ministry of Health and Population, Cairo, Egypt; ${ }^{8}$ Faculty of Clinical Pharmacy, Fayoum University, Fayoum, Egypt; ${ }^{9}$ Department of Clinical Product Development, Institute of Tropical Medicine (NEKKEN), School of Tropical Medicine and Global Health, Nagasaki University, Nagasaki, Japan

\subsection{6/archdischild-2019-epa.296}

Background Neonatal jaundice is expected to be seen between $(60-80) \%$ of healthy newborns. Massage therapy has been practiced for centuries to gain health benefits in infants. Our aim is to evaluate the effectiveness of various types of infant massage in the reduction of neonatal jaundice and to decrease the need for phototherapy (PT).

Methods An electronic databases search was conducted, with inclusion of studies that investigated newborn babies receiving massage therapy with documented total bilirubin before and after the treatment. Study protocol was registered on PROSPERO, CRD42016049025. Quality assessment was performed using NIH risk of bias tool. Frequentist network meta-analysis (NMA) and meta-analysis (MA) were used to compare all outcomes by each day of follow-up.

Results With 690 studies initially identified, 32 were eligible for qualitative analysis and 29 for quantitative meta-analysis. For neonates requiring PT, our NMA results showed that in 3rd day of life, massage and phototherapy (MP) was significantly the most effective in reduction of serum bilirubin level, while acupressure massage and phototherapy (AMP) was the least compared to by phototherapy only (P), with $\mathrm{MD}, 95 \%$ CI $(-1.16$ [-1.61; -0.72$], 1.28[0.79 ; 1.77]$ respectively). In 14 th day of life, bilirubin level decreased the most in massage with enema and phototherapy (MEP), while MP has no significant difference compared to P. Our MA results in 4th day of life, indicated that MP had a significant decrease in bilirubin level compared with $\mathrm{P}$, with MD 95\%CI (-2.23 [-3.43; 1.03]). In summary comparison with $P, M P$ was the most in decreasing bilirubin level in most of days, with the biggest decrease being in 4th day, while MEP was significantly the most in 14th day. For neonates not requiring PT, our NMA showed a significant decrease in bilirubin with massage and bathing, acupressure massage, and massage 3-4 times/days (M2) groups in 3rd day of life, and with massage 1-2 times/ days (M1) and M2 groups in 4th day. MEP group had a significantly longer duration of PT compared with P, with MD 95\%CI $(29.09$ [9.76; 48.41]). M1 and M2 did not have a significant effect on the requirement for PT. 